\title{
Establishing the chromatographic fingerprints of flavan-3-ols and proanthocyanidins from rose hip (Rosa sp.) species
}

\author{
Dragana Č. Dabić Zagorac ${ }^{1}$ (iD) | Milica M. Fotirić Akšić ${ }^{2}$ ｜ Vesna Glavnik ${ }^{3}$ | \\ Uroš M. Gašićc ${ }^{4}$ | Irena Vovk ${ }^{3} \quad$ Živoslav Lj. Tešićc $\quad$ | Maja M. Natićc
}

${ }^{1}$ Innovation centre of the Faculty of Chemistry, University of Belgrade, Belgrade, Serbia

${ }^{2}$ Faculty of Agriculture, University of Belgrade, Zemun, Serbia

${ }^{3}$ Department of Food Chemistry, National Institute of Chemistry, Ljubljana, Slovenia

${ }^{4}$ Institute for Biological Research "Siniša Stanković" - National Institute of Republic of Serbia, University of Belgrade, Belgrade, Serbia

${ }^{5}$ Faculty of Chemistry, University of Belgrade, Belgrade, Serbia

\section{Correspondence}

Dragana DabićZagorac, Innovation centre of the Faculty of Chemistry, University of Belgrade, Studentski trg 12-16, 11000

Belgrade, Serbia.

Email: ddabic@chem.bg.ac.rs
The profile of flavan-3-ols and proanthocyanidins in five different Rosa species ( $R$. canina, $R$. glutinosa, $R$. rubiginosa, $R$. multiflora, and $R$. spinosissima) was estimated on high performance thin layer chromatography cellulose plates. Differences in flavanol and proanthocyanidin profiles of the extracts were evident, among which Rosa spinosissima stood out with catechin as the only detected flavanol and red zones as indication of anthocyanins. Furthermore, the elution solvent for thin layer chromatography with mass spectrometry analyses of glycosylated flavan-3-ols and proanthocyanidins was optimized, enabling identification of catechin, (epi)catechin hexoside, proanthocyanidin dimer, and proanthocyanidin dimers and trimers hexosides. A total of 15 flavanols and their derivatives were identified using ultra-highperformance liquid chromatography with linear trap quadrupole-Orbitrap mass analyzer and epicatechin, gallocatechin, and proanthocyanidin trimer were identified only using this technique. However, proanthocyanidin trimer trihexoside was identified only by thin-layer chromatography with mass spectrometry. To establish the relationships between the flavanols and proanthocyanidins composition of rose hip and their origin, principal component analysis was performed on the entire set of liquid chromatography/mass spectrometry data. Both principal components' scores plots showed that Rosa spinosissima could be considered as an outlier. Our study demonstrated that flavanol and proanthocyanidin profiles of different rose hips depend on the geographical origin rather than on the cultivar and genotype.

\section{K E Y W O R D S}

fingerprint, flavanol, proanthocyanidins, rose hip, thin-layer chromatography

\section{1 | INTRODUCTION}

The genus Rosa contains over 100 species that are widely distributed mostly in Europe, Asia, the Middle East, and North

Article Related Abbreviations: DMACA, 4-dimethylaminocinnamaldehyde; HPTLC, high-performance thin-layer chromatography; LTQ-Orbitrap MS ${ }^{\mathrm{n}}$, linear trap quadrupole-Orbitrap mass analyzer; PA, proanthocyanidin; PC, principal component; PCA, principal component analysis.
America [1]. The rose hip is the pseudo fruit of plants belonging to Rosa genus. The usage of those medicinal plants dates back to Hippocrates' time. During World War II, it was used for preventing scurvy since it was rich in vitamin C [2]. In traditional folk medicine, aqueous extracts of petals, fruit, and leaves of Rosa plants are applied to treat various diseases such as nephritis, common cold, flu, coughing, bronchitis, eczema, itching, and biliary diseases [3,4]. Rose hips are endowed with vitaminisant, astringent, colagogue, choleretic, diuretic, antidiarrhoea, and antioxidant properties. The rose hips are 
also considered as an important source of food [5]. Roses are economically the most important ornamental plants and are also used as a source of aromatic oils for the perfume industry [6].

Studies on the phytochemical compounds found in Rosa species have shown that its fruits contain phenolic acids [7], proanthocyanidins (PAs) and pectins [3], flavonoids [8], fatty acids [9], fruit acids (ascorbic acid, malic acid, and citric acid) [10], essential oils [11], phyto-oestrogens [12], sugars and vitamin C [13], minerals [14], and carotenoids [15]. Clinical studies have shown that polyphenols found in large amounts in rose hip extracts, are responsible for the antioxidant, antiinflammatory, and antimutagen effect $[16,17]$. Simplicity, inexpensive operating costs, and simultaneous analysis of several samples and standards, put high-performance thinlayer chromatography (HPTLC) in a favourable position in certain investigations [18-20]. HPTLC was applied for both qualitative [21-25] and quantitative analyses of flavanols in plant extracts [26-30]. A significant contribution to the analysis of flavan-3-ol and PAs using HPTLC-MS in combination of silica gel, diol, and cellulose was given so far [31-33] especially when two-step predevelopment procedure solution for high backgrounds and ion suppression effects was proposed on silica gel [31]. The aim of this study was: (i) to assess flavan-3-ol and PA profiles of rose hip extracts of five Rosa species: Rosa canina, Rosa glutinosa, Rosa rubiginosa, Rosa multiflora, and Rosa spinosissima by using HPTLC coupled to MS and UHPLC coupled with linear trap quadrupole and Orbitrap mass analyzer (UHPLC-LTQ-Orbitrap $\mathrm{MS}^{\mathrm{n}}$ ), (ii) to propose tentative structures of glycosylated flavan-3-ols and PAs on the basis of their monoisotopic mass and MS/MS fragmentation, and (iii) to show whether it is possible to obtain information on specifics of Rosa species and/or their geographical origin based on the flavan-3-ol and PA profiles, using principal component analysis (PCA).

\section{2 | MATERIALS AND METHODS}

\section{1 | Plant material}

The high yielding dog rose genotypes were selected from the spontaneous flora (from populations that showed no anthropogenic influence) and collected for experiments from diverse locations (Supporting Information Table S1). The genotypes were identified according to taxonomical criteria [34] as $R$. canina (ten genotypes), $R$. glutinosa (one genotype), $R$. rubiginosa (one genotype), $R$. multiflora (one genotype), and $R$. spinosissima (one genotype). All selected bushes were wellformed, without any pest symptoms. Thirty mature hips per genotype were harvested randomly according to shape and color uniformity, from all cardinally-oriented branches with different directions around the bush. Selected rose hips were harvested at the fully ripe maturity stage for each species as judged by their colour [35]. After a short transport in cold containers, samples were stored at $-20^{\circ} \mathrm{C}$ until chemical analysis.

\section{2 | Reagents and standards}

Acetonitrile and methanol (both of MS grade) were purchased from Fluka (Buch, Switzerland). Toluene, formic acid, acetic acid, and 4-dimethylaminocinnamaldehyde (DMACA) were obtained from Merck (Darmstadt, Germany), and methanol and $n$-propanol (both of HPLC grade) from SigmaAldrich (Steinheim, Germany). Standard of (+)-catechin was obtained from Carl Roth (Karlsruhe, Germany), (-)epicatechin and rutin from Sigma-Aldrich, and procyanidin $\mathrm{B} 1$, procyanidin $\mathrm{B} 2$, procyanidin $\mathrm{A} 2$, epicatechin gallate, and (-)-epigallocatechin gallate from Extrasynthesè (Genay, France). All other reagents were of analytical grade. Bidistilled water was used.

\section{3 | Preparation of rose hip extracts and standard solutions}

Rose hip samples, frozen with liquid nitrogen, were ground up by Mikro-Dismembrator S (Sartorius, Göttingen, Germany) at a frequency of 1700/min for $1 \mathrm{~min}$. Each sample $(500 \mathrm{mg}$ ) was mixed with $10 \mathrm{~mL}$ of $70 \%$ methanol and stirred for $10 \mathrm{~min}$ on a magnetic stirrer. After centrifugation at frequency of $4000 \mathrm{rpm}$ for $5 \mathrm{~min}$, the supernatant was filtered through a $0.45 \mu \mathrm{m}$ polyvinylidene fluoride membrane filter (Millipore, Billerica, MA, USA) and stored at $-20^{\circ} \mathrm{C}$ prior to analysis. All extractions were done in triplicate. All stock solutions of flavan-3-ol and PA standards used for HPTLC and HPTLCMS analysis were prepared in methanol with concentration of $100 \mu \mathrm{g} / \mathrm{L}$. A $1000 \mathrm{mg} / \mathrm{L}$ stock solution of catechin standard was used for UHPLC-LTQ-Orbitrap MS ${ }^{\mathrm{n}}$ analysis. All solutions were stored in a refrigerator at $-80^{\circ} \mathrm{C}$.

\section{4 | HPTLC}

Separations were performed on $20 \mathrm{~cm} \times 10 \mathrm{~cm}$ HPTLC cellulose plates (Merck, Darmstadt, Germany). Before application, the plates were predeveloped in water [36]. Rose hip extracts $(10 \mu \mathrm{L})$ and standard solutions (application volumes are given in each figure caption) were applied as $8 \mathrm{~mm}$ bands, $10 \mathrm{~mm}$ from the bottom of the plate, and $15 \mathrm{~mm}$ from the left edge of the plate using an application device Automatic TLC Sampler 4 (Camag, Muttenz, Switzerland). Plates were developed up to $80 \mathrm{~mm}$ with pure water [24,25], $n$-propanol/water/acetic acid $(20: 80: 1, \mathrm{v} / \mathrm{v} / \mathrm{v})$ [25] or $n$-propanol/water/acetic acid $(4: 2: 1, \mathrm{v} / \mathrm{v} / \mathrm{v})[25]$ as developing solvents in an unsaturated twin trough chamber (Camag). After development the plates were dried in a stream of warm air for $2 \mathrm{~min}$, followed by postchromatographic derivatization with DMACA reagent. Specific details of the postchromatographic derivatization 
can be seen in Supporting Information. The chromatographic plates were captured 10 min after derivatization under UV $(366 \mathrm{~nm})$ and white light by using DigiStore 2 Documentation System (Camag).

\section{5 | HPTLC with MS detection}

HPTLC cellulose plates were cut into $10 \mathrm{~cm} \times 10 \mathrm{~cm}$, $7 \times 10 \mathrm{~cm}$, or $6 \times 10 \mathrm{~cm}$ and $n$-propanol/water/acetic acid $(4: 2: 1, \mathrm{v} / \mathrm{v} / \mathrm{v})$ was used as the developing solvent. Application volumes of rose hip extracts were $15 \mu \mathrm{L}$. To achieve better resolution for samples 1-3, water was used as a mobile phase. Rose hip extracts were applied as $6 \mathrm{~mm}$ bands, $10 \mathrm{~mm}$ or $15 \mathrm{~mm}$ from the bottom of the plate, and $15 \mathrm{~mm}$ from the left edge of the plate. After development and drying, only the first sample track was derivatized with DMACA detection reagent (Supporting Information Fig. S1A). The blue zones visible on the derivatized track were used for proper positioning of elution head of TLC-MS interface (Camag) and each zone was eluted from the plate and transferred into the MS detector. The whole plates were dipped into DMACA reagent after HPTLCMS analysis, (Supporting Information Fig. S1B). Additional information of HPTLC-MS analysis can be seen in Supporting Information.

\subsection{UHPLC coupled with linear ion trap-Orbitrap MS}

In order to identify flavan-3-ols and PAs in the negative ionization mode, an LC system consisting of a quaternary pump 600 Accela and Accela Autosampler connected to a linear trap quadrupole Orbitrap mass spectrometer with ESI-heated probe (HESI-II, ThermoFisher Scientific, Bremen, Germany) was used. Separation was performed on a Syncronis C18 column $(100 \mathrm{~mm} \times 2.1 \mathrm{~mm}, 1.9 \mu \mathrm{m})$ column with mobile phase consisting of (A) water $+0.1 \%$ formic acid and (B) acetonitrile $+0.1 \%$ formic acid. A linear gradient program: 0.0 $1.0 \mathrm{~min} 5 \%$ (B), $1.0-12.0 \mathrm{~min}$ from 5 to $95 \%$ (B), 12.0 $12.2 \mathrm{~min}$ from 95 to $5 \%(\mathrm{~B})$, and then $5 \%$ (B) for 3 min were used [37]. Ion source settings and parameters were the same as in the study of Natić et al. [37]. Xcalibur software (version 2.1) was used for instrument control and data analysis. Molecule editor program, ChemDraw (version 12.0) was used as to calculate accurate mass of compounds of interest. Unknown compounds were identified on the basis of their monoisotopic mass and $\mathrm{MS}^{4}$ fragmentation, and confirmed using previously reported MS fragmentation data found in literature $[38,39]$.

\section{7 | PCA}

PCA was realized using the PLS_Tool Box software package for MATLAB (Version 7.12.0). All data were group scaled prior to PCA. The singular value decomposition algorithm

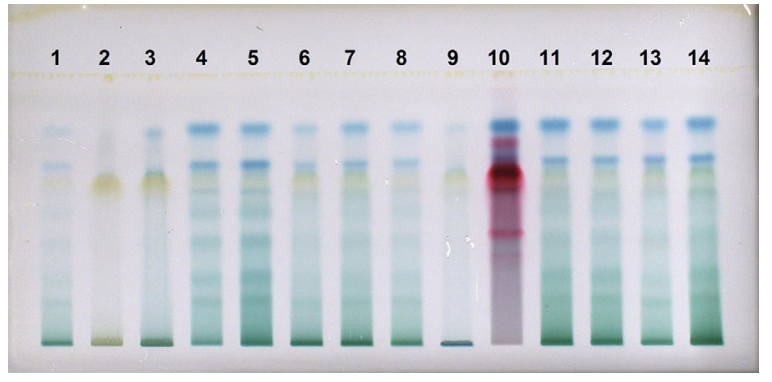

F I G U R E 1 Chromatograms of 14 rose hip extracts (Supporting Information Table S1) on HPTLC cellulose plates developed using $n$-propanol/water/acetic acid (4:2:1, v/v/v)

and a 0.95 confidence level for $Q$ and Hotelling's $T^{2}$ limits for outliers were chosen.

\section{3 | RESULTS AND DISCUSSION}

\section{1 । HPTLC analysis of rose hip extracts}

Flavan-3-ols and PAs profiles of rose hips extracts were investigated using HPTLC cellulose plates developed in: pure water, $n$-propanol/water/acetic acid (20:80:1, v/v/v), and $n /$ propanol/water/acetic acid (4:2:1, v/v/v; Supporting Information Fig. S2). The obtained chromatogram showed relatively good resolution, but separation was not satisfactory (Supporting Information Fig. S2A). When mixture of $n$ propanol/water/acetic acid (20:80:1, v/v/v) was used as a developing solvent, both resolution and separation were not satisfactory (Supporting Information Fig. S2B). Finally, a mixture of $n$-propanol/water/acetic acid $(4: 2: 1, \mathrm{v} / \mathrm{v} / \mathrm{v})$ proved to be the most appropriate developing solvent (Supporting Information Fig. S2C): A chromatogram with the largest number of zones in the tracks of the samples was obtained and the zones were the most compact.

The chromatograms developed using a mixture of $n$-propanol/water/acetic acid $(4: 2: 1, \mathrm{v} / \mathrm{v} / \mathrm{v})$ are shown in Fig. 1. Differences among flavan-3-ols and PA profiles of rose hips extracts are evident. All samples, with the exception of sample 2, contained the zone with the highest $R_{\mathrm{F}}$ value, which corresponded to catechin standard (Supporting Information Fig. S2C). The epimers, (+)-catechin and (-)-epicatechin are separated using $n$-propanol/water/acetic acid $(4: 2: 1, \mathrm{v} / \mathrm{v} / \mathrm{v})$ as developing solvent [25]. However, complete characterization of the compound corresponding to the blue colored zone with the highest $R_{\mathrm{F}}$ value in sample 9 was only possible using TLC-MS analysis. Sample $10(R$. spinosissima) with the most intense zone with the highest $R_{\mathrm{F}}$ value and red/pink colored zones that indicate the presence of anthocyanins proved to be a bit different compared to the other investigated rose hip samples. Number and intensity of blue colored zones in the chromatograms of the extracts 2, 3, 9 , and 10 is lower than in other extracts. 


\section{2 | HPTLC-MS coupling and optimization of MS conditions}

The eluent selection and optimization of elution conditions is an important step in the HPTLC-MS analysis. Following procedure described by Smrke and Vovk [33], pure methanol and mixture methanol/acetonitrile $(1: 2, \mathrm{v} / \mathrm{v})$ were primarily used as eluents in HPTLC-MS analysis of flavan-3-ol and PA profiles of rose hip extracts. However, the two eluents were inadequate for MS analysis of glycosylated flavan-3-ols and PAs, showing a high level of noise in MS spectra (Supporting Information Fig. S3A and B). Moreover, HPTLC cellulose plate dipped into DMACA reagent after HPTLC-MS analysis showed that glycosylated flavan-3-ols and PAs were not eluted completely (the blue colored zones were still visible, Supporting Information Fig. S3C and D). Also, the quality of the data obtained by HPTLC-MS can be hampered by a severe spectral background or by strong ion suppression. This problem was solved with silica-gel using two predevelopments [31]. All these point to the necessity of optimization of HPTLC-MS method in the sense of choosing the most appropriate eluent.

Here, in order to find an optimal system for elution of glycosylated flavan-3-ols and PAs, four different eluents were used: 1$)$ methanol; 2) acetonitrile/water $(2: 1, \mathrm{v} / \mathrm{v}) ; 3)$ methanol/water $(7: 3, \mathrm{v} / \mathrm{v})$; and 4$)$ acetonitrile/water $(2: 1, \mathrm{v} / \mathrm{v}$; $1 \%$ formic acid in acetonitrile was added to the effluent prior to injection into the liquid chromatography quadrupole (LCQ) system). For the first three eluents, $1 \%$ formic acid in methanol was added to the effluent prior to injection into the LCQ system. HPTLC cellulose plate derivatized after HPTLC-MS (Supporting Information Fig. S4) showed which eluents were effective in elution. Namely, methanol was not an appropriate eluent for the investigation of flavan-3-ols and PAs on cellulose (zones were still blue colored after HPTLCMS analysis). As an example, MS spectra of PA trimer dihexoside $m / z, 1189$ (zone F, Supporting Information Fig. S4) and PA trimer trihexoside $\mathrm{m} / \mathrm{z} 1351$ (zone G, Supporting Information Fig. S4) are presented in Supporting Information Fig. S5. The most appropriate mixtures for MS analysis were methanol/water $(7: 3, \mathrm{v} / \mathrm{v})$ and acetonitrile/water $(2: 1, \mathrm{v} / \mathrm{v})$ with the addition of $1 \%$ formic acid in methanol to the effluent prior to injection into the LCQ system. The intensity of peaks was higher when methanol/water $(7: 3, \mathrm{v} / \mathrm{v})$ was used. However, using mixture methanol/water $(7: 3, \mathrm{v} / \mathrm{v})$ resulted in a high pressure ( $\sim 30-35$ bars) in the system, which led to a rapid clogging of the interface, and this was the reason for choosing acetonitrile/water (2:1, v/v) for HPTLC-MS analysis.

\section{3 | Identification of flavan-3-ols and PAs in rose hip extracts by TLC-MS}

Each rose hip extract was analyzed on separate HPTLC cellulose plates in four equal applications $(15.0 \mu \mathrm{L})$. Mix- ture of $n$-propanol/water/acetic acid $(4: 2: 1, \mathrm{v} / \mathrm{v} / \mathrm{v})$ used as developing solvent proved to be inadequate for the analysis of the extracts 1,2, and 3 (Supporting Information Table S1, Fig. S6), so pure water was used as mobile phase. Results of HPTLC-MS analyses are summarized in Table 1 and HPTLC-MS spectrum of sample 5 is given in Fig. 2 as an example. The total intensities obtained from the MS/MS spectra for all identified compounds are presented in Supporting Information Table S3. A total of seven flavan-3-ol derivatives were identified based on the search for the $[\mathrm{M}-\mathrm{H}]^{-}$ deprotonated molecule and its $\mathrm{MS}^{2}$ and $\mathrm{MS}^{3}$ fragmentations. All identified compounds were found in ten of fourteen investigated samples. All flavan-3-ol derivatives gave molecular ions in the form of $[\mathrm{M}-\mathrm{H}]^{-}$deprotonated molecules, except (epi)catechin hexoside-chloride $(487 \mathrm{~m} / \mathrm{z}$; Table S2), which was ionized in the form of adduct with $\mathrm{HCl}$. In $\mathrm{MS}^{2}$ spectrum of (epi)catechin hexoside-chloride, base peak fragment at 451 $\mathrm{m} / \mathrm{z}$ (deprotonated mass of (epi)catechin hexoside) and secondary $\mathrm{MS}^{2}$ peak of high intensity at $289 \mathrm{~m} / \mathrm{z}$ resulting from loss of hexose (162 Da) can be observed. Furthermore, profiles of samples 1, 2, 3, 9, and 10 were poorer when compared with other rose hip extracts. Extracts 2 and 9 contained only PA dimer, while in the extract 3 catechin was found along with PA dimer. Based on the chromatogram presented in Fig. 1, it could be assumed that all samples, with exception of sample 2 , contained catechin. However, HPTLC-MS analyses showed the absence of catechin in the extract 9, because the zone with the highest $R_{\mathrm{F}}$ value (zone I, Supporting Information Fig. S7) corresponded to the mass $609 \mathrm{~m} / \mathrm{z}$ (Supporting Information Fig. S7), not $289 \mathrm{~m} / \mathrm{z}$, which would be in the case of catechin. At first glance, insight into MS fragmentation of this compound at $609 \mathrm{~m} / \mathrm{z}$, it was considered to be a rutin or its isomer, but the final structure elucidation of it was confirmed later using high resolution MS. Furthermore, sample 10 proved to be a bit different when compared to the other rose hip samples, as it contained anthocyanins (red zones, Fig. 1) and catechin was only detected flavan-3-ol.

\section{4 | Determination of flavan-3-ols and PAs by HPLC-MS}

A total of 15 flavanols and PAs were identified based on the search for the $[\mathrm{M}-\mathrm{H}]^{-}$deprotonated molecule and its $\mathrm{MS}^{4}$ fragmentation. Chromatographic and MS data are summarized in Supporting Information Table S4. Peak identification was based on published data. The total intensities (peak areas) for all identified compounds were obtained from the full scan spectra (Supporting Information Table S5). In analyzed extracts, it was possible to identify flavan-3-ol monomers (catechin, epicatechin, and gallocatechin) and their derivatives (PA dimers, trimers and their hexosides). Presence of flavan3-ol monomers was confirmed using available standards. As for PA dimers, four of them were identified giving $\mathrm{MS}^{2}$ base 


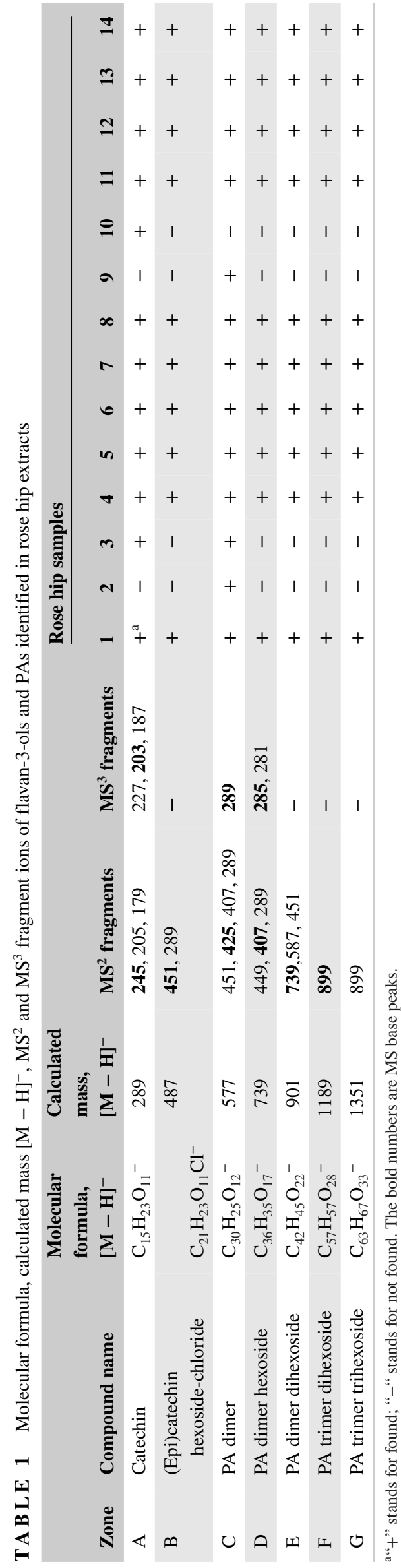

peak at $425 \mathrm{~m} / \mathrm{z}$ and secondary $\mathrm{MS}^{2}$ peaks at 451,407 , and $289 \mathrm{~m} / \mathrm{z}$. Compound with molecular ion at $451 \mathrm{~m} / \mathrm{z}, \mathrm{MS}^{2}$ base peak at $137 \mathrm{~m} / z$, and secondary $\mathrm{MS}^{2}$ peaks at 289 and 271 $\mathrm{m} / \mathrm{z}$ was tentatively identified as (epi)catechin-hexoside. It was interesting at this site to define a compound at $609 \mathrm{~m} / \mathrm{z}$, which was found to be main peak in the rose hip extract $9(R$. multiflora). It produced the $\mathrm{MS}^{2}$ base peak at $301 \mathrm{~m} / z$ and secondary $\mathrm{MS}^{2}$ peak at $447 \mathrm{~m} / \mathrm{z}$, which was formed by loss of hexose unit of $162 \mathrm{Da}$. From these data, it was concluded that this compound was an isomer of rutin (quercetin 3-Orutinoside); because it had the same exact mass like rutin, but it differs in the $\mathrm{MS}^{2}$ spectrum $\left(\mathrm{MS}^{2}\right.$ secondary peak in the case of rutin would be at $463 \mathrm{~m} / \mathrm{z}$, corresponding to the loss of rhamnose unit of $146 \mathrm{Da}$ ). This was confirmed by recording of rutin standard. Using well-known rules for determining interglycosidic linkage in the case of flavonoid glycosides [40] and based on MS fragmentation, this compound was marked as quercetin 3-O-(4'-hexosyl)rhamnoside. By searching the available literature on flavonoids in different $R$. spinosissima, it was concluded that this compound was already isolated and identified in R. multiflora [41] by the name multinoside.

\section{5 | Comparison of flavan-3-ol PAs profiles of rose hip extracts}

PCA was performed separately on the results obtained by HPTLC-MS and UHPLC-LTQ-Orbitrap MS ${ }^{\mathrm{n}}$ (Fig. 3). PCA of HPTLC-MS data resulted in three principal components (PCs) explaining $96.1 \%$ of the variation of the data set. PC1 accounted for $73.8 \%$, PC2 $17.5 \%$, and PC3 $4.9 \%$ of the total variance. As for the results of UHPLC-LTQ-Orbitrap MS ${ }^{\mathrm{n}}$, the first four components explained $93.8 \%$ of the total variance (PC1, PC2, PC3, and PC4 accounted for 45.7, 31.1, 12.4, and $4.6 \%$, respectively). Although the number of flavanols and PAs identified by UHPLC-LTQ-Orbitrap MS ${ }^{\mathrm{n}}$ was twice as high as by HPTLC-MS, similar clustering on PC scores plots was obtained (Fig. 3A and C). Both PC scores plots showed that sample 10 (R. spinosissima, Uvac) exceeded the limits imposed by the Hotelling's $T^{2} 95 \%$ probability ellipse and could be considered as an outlier. Loadings plot based on HPTLC-MS results revealed that catechin discriminated sample 10 from the other samples (Fig. 3B). Gallocatechin (I), PA dimer isomer 1(XII), and epicatechin (XIII) were identified by UHPLC-LTQ-Orbitrap $\mathrm{MS}^{\mathrm{n}}$ only in extract of $R$. spinosissima (sample 10) and had the highest impact on separation of the sample 10 (Fig. 3D).

PCA applied on HPTLC-MS results showed clustering of samples from location Gornji Milanovac (samples 11-14) along the PC2 dimension (Fig. 3A). Higher levels (the total intensities) of PA dimer, PA dimer hexoside, PA dimer dihexoside, PA trimer dihexoside, and PA trimer trihexoside were the most influential in separation of samples from location Gornji Milanovac among the rest of samples (Fig. 3B). Additionally, 


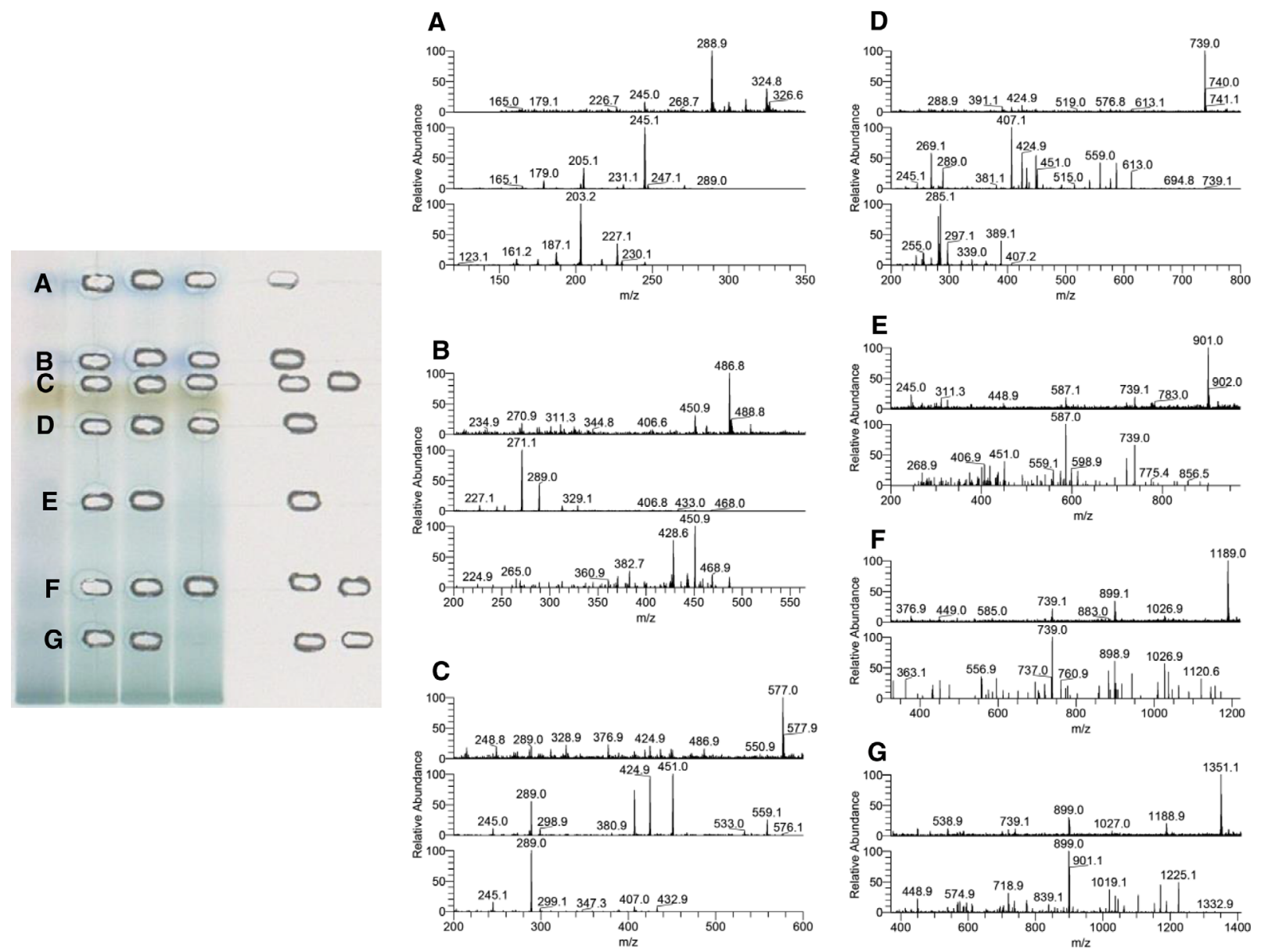

F I G U R E 2 MS spectra of rose hip extract 5 eluted with acetonitrile/water (2:1, v/v) from HPTLC cellulose plate. A) catechin; B) (epi)catechin hexoside-chloride; C) PA dimer B type; D) PA dimer monohexoside; E) PA dimer dihexoside; F) PA trimer dihexoside; G) PA trimer trihexoside

samples 4 and 5 ( $R$. glutinosa and $R$. rubiginosa, respectively) from location Mavrovo were distinguished by their high level of (epi)catechin hexoside-chloride. Samples 2 and 3 (both $R$. canina) from Novi Sad and sample 9 ( $R$. multiflora) from Vršac were grouped according to poorer flavanols and PAs profile, where only PA dimer was identified in samples 2 and 9. PCA based on UHPLC-LTQ-Orbitrap MS ${ }^{\mathrm{n}}$ data resulted in PC scores plot (Fig. 3C) where separation according to geographical origin was worse in comparison with PCA applied on HPTLC-MS results. Samples 1-3 from Novi Sad, together with 6 and 9 from Vršac, were separated from the other samples along the PC1. PAs (II-V, VII, and X) had the highest positive impact on $\mathrm{PC} 1$ and were the most influential in distinguishing samples 1, 2, 3, 6, and 9 from the other samples.

\section{4 | CONCLUDING REMARKS}

The high-resolution MS as a powerful technique allowed the identification of characteristic flavanols derivatives. Optimization of elution conditions and selection of eluent proved to be an important step in HPTLC-MS analysis. Mixture of acetonitrile/water (2:1, v/v; $1 \%$ formic acid in methanol was added to the effluent prior to injection into the LCQ system) was chosen as the most suitable one. A total of seven flavanols derivatives, flavanol monomers, PA dimmers, trimers, and their hexosides, were identified by HPTLC-MS. The number of identified flavanol derivatives in several extracts, such as two $R$. canina samples from Novi Sad, $R$. multiflora from Vršac, and $R$. spinosissima from Uvac, were significantly lower when compared with the other investigated samples. Catechin was detected in all investigated rose hip extracts with the exception of $R$. canina (Novi Sad) and $R$. multiflora (Vršac). Furthermore, only PA dimer was detected in these samples. On the other hand, the highest content of catechin was found in $R$. spinosissima (Uvac). Also, UHPLC-LTQOrbitrap $\mathrm{MS}^{\mathrm{n}}$ was used to determine flavanol and PA profiles of rose hip extracts. The number of flavanol derivatives identified by UHPLC-LTQ-Orbitrap $\mathrm{MS}^{\mathrm{n}}$ was twice as higher while using HPTLC-MS mostly due to the fact that several isomers of the PA dimer, trimer, and their hexosides were identified. Actually, gallocatechin, PA trimer, and epicatechin were identified only using UHPLC-LTQ-Orbitrap $\mathrm{MS}^{\mathrm{n}}$. On the other hand, PA trimer trihexoside was identified in rose hip 

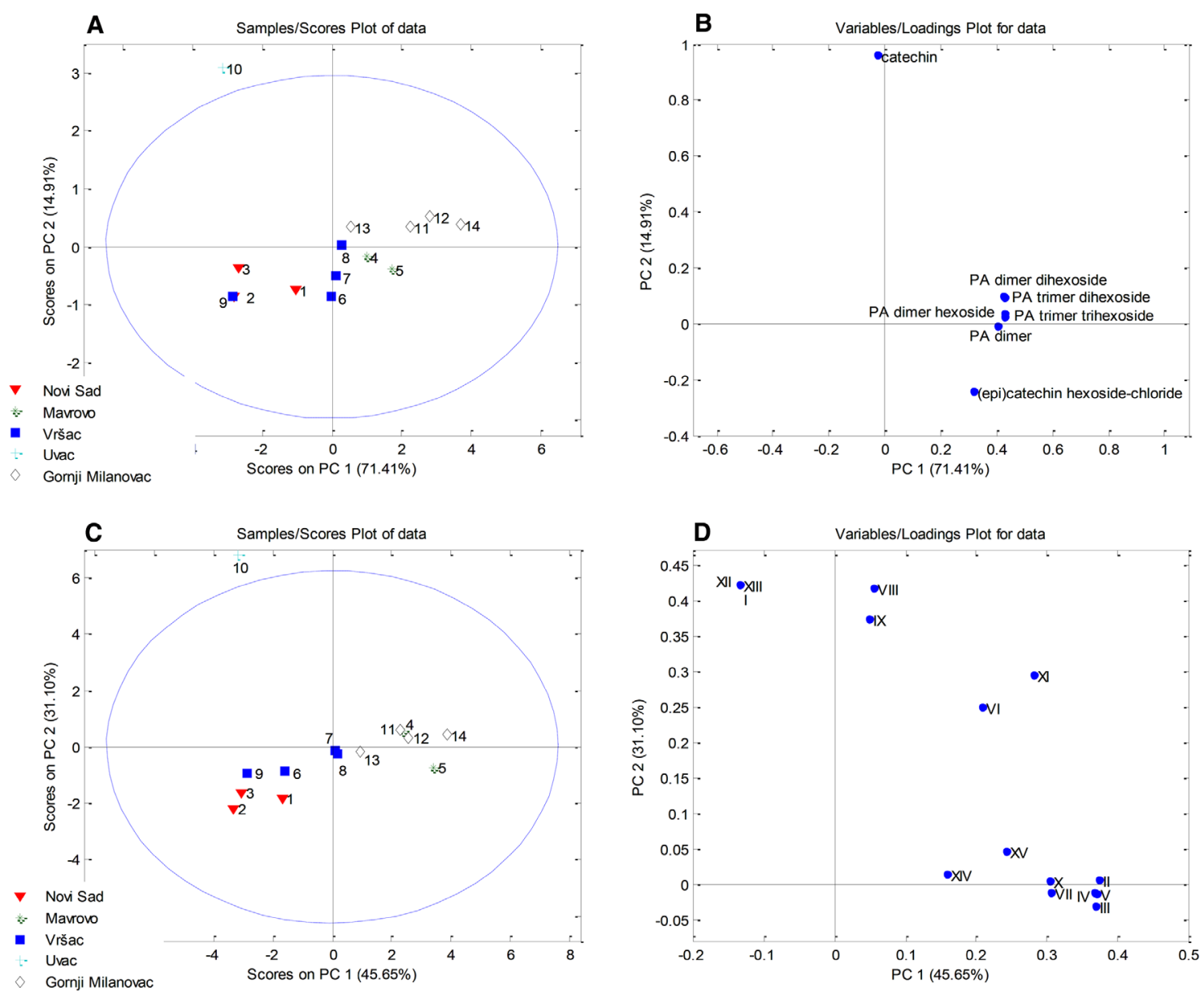

F I G U R E 3 PC scores (A) and loadings (B) plot of results obtained by HPTLC-MS; PC scores (C) and loadings (D) plot of results obtained by UHPLC-LTQ-Orbitrap MS ${ }^{\mathrm{n}}$. Numbers on Figure 3D correspond to the detected flavanol derivatives as given in Supporting Information Table S4

extracts only by HPTLC-MS. A multivariate analysis, PCA employed on the entire set of semiquantitative data revealed from chromatogram in LC/MS experiment, showed a clustering of samples in two clusters. Such a grouping was a consequence of the difference in flavanol and PA profiles, where variations in the content of flavanol derivatives were designated as important parameters for the discrimination of the samples. This research provided an answer to the question whether the flavanol and PA profiles of different rose hips depend on the geographical origin rather than on the cultivar and genotype. Finally, similar profiles that were obtained demonstrated the usefulness of the HPTLC-MS as it was not only sufficiently reliable but could also be the method of choice when analyzing flavanols and PAs containing taxa, as it is easy to operate, economical with low consumption of samples and solvents, and based on the green chemistry principles and practices.

\section{ACKNOWLEDGMENTS}

The research was performed in the Department of Food Chemistry at the National Institute of Chemistry supported from the Slovenian Research Agency (No. P1-0005) and ENFIST Centre of Excellence infrastructure (TLC-MS interface) and by the Ministry of Education, Science and Technological Development, Republic of Serbia (No. TR 31063 and No. 172017).

\section{CONFLICT OF INTEREST}

The authors declare no potential conflict of interest.

\section{ORCID}

Dragana $\check{C}$. Dabić Zagorac (iD

https://orcid.org/0000-0001-5454-5696

\section{REFERENCES}

1. Nilsson, O., Rosa, in: Davids, P. H. (Ed.), Flora of Turkey and the East Aegean Islands. Edinburgh University Press, Edinburgh 1997, pp. 106-128.

2. Haas, L.F., Rosa canina (dog rose). J. Neurol. Neurosurg. Psychiatry 1995, 59, 470. 
3. Chrubasik, C., Roufogalis, B. D., Müller-Ladner, U., Chrubasik, S. A systematic review on the Rosa canina effect and efficacy profiles. Phytother. Res. 2008, 22, 725-733.

4. Kultur, S., Medicinal plants used in Kırklareli Province (Turkey). $J$. Ethnopharmacol. 2007, 111, 341-364.

5. Demir, F., Özcan, M., Chemical and technological properties of rose (Rosa canina L.) fruits grown wild in Turkey. J. Food Eng. 2001, 47, 333-336.

6. Gudin, S., Rose: Genetics and breeding. Plant Breed. Rev. 2000, 17, 159-189.

7. Hvattum, E., Determination of phenolic compounds in rose hip (Rosa canina) using liquid chromatography coupled to electrospray ionisation tandem mass spectrometry and diode-array detection. Rapid Commun. Mass Spectrom. 2002, 16, 655-662.

8. Kumarasamy, Y., Cox, P. J., Jaspars, M., Rashid, M. A., Sarker, D. S., Bioactive flavonoid glycosides from the seeds of Rosa canina. Pharm. Biol. 2003, 41, 237-242.

9. Koponen, J. M., Happonen, A. M., Mattila, P. H., Torronen, A. R., Contents of anthocyanins and ellagitannins in selected foods consumed in Finland. J. Agric. Food Chem. 2007, 55, 1612-1619.

10. Nowak, R., Chemical composition of hips essential oils of some Rosa L. species. Z. Naturforsch. C J. Biosci. 2005, 60, 369-378.

11. Valsta, L. M., Kilkkinen, A., Mazur, W., Nurmi, T., Lampi, A. M., Ovaskainen, M. L., Korhonen, T., Adlercreutz, H., Pietinen, P., Phyto-oestrogen database of foods and average intake in Finland. Br. J. Nutr. 2003, 89, S31-S38.

12. Uggla, M., Gao, X., Werlemark, G., Variation among and within dogrose taxa (Rosa sect. caninae) in fruit weight, percentages of fruit flesh and dry matter, and vitamin C content. Acta Agric. Scand. B 2003, 53, 147-155.

13. Basgel, S., Erdemoglu, S. B., Determination of mineral and trace elements in some medicinal herbs and their infusions consumed in Turkey. Sci. Total Environ. 2006, 359, 82-89.

14. Bohm, V., Frohlich, K., Bitsch, R., Rose hip - a "new" source of lycopene? Mol. Aspects Med. 2003, 24, 385-389.

15. Larsen, E., Kharazmi, A., Christensen, L. P., Christensen, S. B. An antiinflammatory galactolipid from rose hip (Rosa canina) that inhibits chemotaxis of human peripheral blood neutrophils in vitro. J. Nat. Prod. 2003, 66, 994-995.

16. Fecka, I., Qualitative and quantitative determination of hydrolysable tannins and other polyphenols in herbal products from meadowsweet and dog rose. Phytochem. Anal. 2009, 20, 177-190.

17. Vovk, I., Albreht, A., TLC-MS analysis of carotenoids, triterpenoids, and flavanols in plant extracts and dietary supplements, in: Kowalska, T., Sajewicz, M., Sherma, J. (Eds.), Planar Chromatography-Mass Spectrometry. CRC Press, Boca Raton, FL 2016, pp. 305-326.

18. Sajewicz, M., Staszek, D., Natić, M., Wojtal, Ł., WaksmundzkaHajnos, M., Kowalska, T., TLC-MS versus TLC-LC-MS fingerprints of herbal extracts. Part II. Phenolic acids and flavonoids. $J$. Liq. Chromatogr. Relat. Technol. 2011, 34, 864-887.

19. Sajewicz, M., Staszek, D., Natić, M., Waksmundzka-Hajnos, M., Kowalska, T., TLC-MS versus TLC-LC-MS fingerprints of herbal extracts. Part III. Application of the reversed-phase liquid chromatography systems with C18 stationary phase. J. Chromatogr. Sci. 2011, 49, 560-567.

20. Glavnik, V., Simonovska, B., Vovk, I., Densitometric determination of (+)-catechin and (-)-epicatechin by 4- dimethylaminocinnamaldehyde reagent. J. Chromatogr. A 2009 , 1216, 4485-4491.

21. Wang, K., Chen, Q., Lin, Y., Yu, S., Lin, H., Huang, J., Liu, Z., Separation of catechins and O-methylated (-)-epigallocatechin gallate using polyamide thin-layer chromatography. J. Chromatogr. B 2016, 1017-1018, 221-225.

22. Phansalkar, R. S., Nam, J. -W., Chen, S. -N., McAlpine, J.B., Napolitano, J. G., Leme, A., Vidal, C. M., Aguiar, T., Bedran-Russo, A. K., Pauli, G. F., A galloylated dimeric proanthocyanidins from grape seed exhibits dentin biomodification potential. Fitoterapia 2015, 101, 169-178.

23. Vovk, I., Simonovska, B., Andrenšek, S., Vuorela, H., Vuorela, P., Rotation planar extraction and rotation planar chromatography of oak (Quercus robur L.) bark. J. Chromatogr. A 2003, 991, 267274.

24. Vovk, I., Simonovska, B., Vuorela, H., Separation of eight selected flavan-3-olson cellulose thin-layer chromatographic plates. J. Chromatogr. A 2005, 1077, 188-194.

25. Boudesocque, L., Dorat, J., Pothier, J., Gueiffier, A., EnguehardGueiffier, C., High performance thin layer chromatographydensitometry: A step further for quality control of cranberry extract. Food Chem. 2013, 139, 866-871.

26. Pobłocka-Olech, L., Krauze-Baranowska, M., SPE-HPTLC of procyanidins from the barks of different species and clones of Salix. J. Pharm. Biomed. Anal. 2008, 48, 965-968.

27. Khan, I., Sangwan, P. L., Abdullah, S. T., Gupta, B. D., Dhar, J. K., Manickavasagar, R., Koul, S., Ten marker compounds-based comparative study of green tea and guava leaf by HPTLC densitometry methods: Antioxidant activity profiling. J. Sep. Sci. 2011, 34, 749760.

28. Rodríguez-Bernaldo de Quirós, A., López-Hernundez, J., FerracesCasais, P., Lage-Yusty, M. A., Analysis of non-anthocyanin phenolic compoundsin wine samples using high performance liquidchromatography with ultraviolet and fluorescencedetection. J. Sep. Sci. 2007, 30, 1262-1266.

29. Glavnik, V., Simonovska, B., Vovk, I., Comparison of TLC and HPLC methods used for analysis of (-)-epicatechin and its dimer procyanidin B2 in chocolate. J. Planar. Chromatogr. 2010, 23, 230 232.

30. Glavnik, V., Vovk, I., Albreht, A., High performance thin-layer chromatography-mass spectrometry of Japanese knotweed flavan3-ols and proanthocyanidins on silica gel plates. J. Chromatogr. A 2017, 1482, 97-108.

31. Glavnik, V., Vovk, I., High performance thin-layer chromatography-mass spectrometry methods on diol stationary phase for the analyses of flavan-3-ols and proanthocyanidins in invasive Japanese knotweed. J. Chromatogr. A 2019, 1598, 196-208.

32. Smrke, S., Vovk, I., Comprehensive thin-layer chromatography mass spectrometry of flavanols from Juniperus communis L. and Punica granatum L. J. Chromatogr. A. 2013, 1289, 119-126.

33. Mratinic, E., Aksic, M. F. The Distribution of Wild Fruit Species in Serbia, LAP Lambert Academic Publishing, Saarbrücken 2019, pp. 1-268.

34. Nojavan, S., Khalilian, F., Kiaie, F., Rahimi, A., Arabanian, A., Chalavi, S., Extraction and quantitative determination of ascorbic acid during different maturity stages of Rosa canina L. fruit. J. Food Compos. Anal. 2008, 21, 300-305. 
35. Vovk, I., Simonovska, B., Vuorela, P., Vuorela, H., Optimization of separation of (+)-catechin and (-)-epicatechin on cellulose TLC plates. J. Planar Chromatogr. 2002, 15, 433-436.

36. Natić, M., Dabić, D., Papetti, A., Fotirić Akšić, M. M., Ognjanov, V., Ljubojević, M., Tešić, Ž., Analysis and characterisation of phytochemicals in mulberry (Morus alba L.) fruits grown in Vojvodina, North Serbia. Food Chem. 2015, 171, 128-136.

37. Rockenbach, I. I., Jungfer, E., Ritter, C., Santiago-Schübel, B., Thiele, B., Fett, R., Galensa, R., Characterization of flavan-3-ols in seeds of grape pomace by CE, HPLC-DAD-MSn and LC-ESIFTICR-MS. Food Res. Int. 2012, 48, 848-855.

38. Lin, L. Z., Sun, J., Chen, P., Monagas, M. J., Harnly, J.M., UHPLC-PDA-ESI/HRMSn profiling method to identify and quantify oligomeric proanthocyanidins in plant products. J. Agric. Food Chem. 2014, 62, 9387-9400.

39. Ferreres, F., Llorach, R., Gil-Izquierdo, A., Characterization of the interglycosidic linkage in di-, tri-, tetra- and pentaglycosylated flavonoids and differentiation of positional isomers by liquid chromatography/electrospray ionization tandem mass spectrometry. J. Mass Spectrom. 2004, 39, 312-21.
40. Norimoto, H., Nakajima, K., Yomoda, S., Morimoto, Y., Testosterone $5 \alpha$-reductase inhibitory constituents from the fruits of Rosa multiflora THUNB. J. Tradit. Med. 2010, 2, 90-95.

\section{SUPPORTING INFORMATION}

Additional supporting information may be found online in the Supporting Information section at the end of the article.

How to cite this article: Dabić Zagorac DČ, Fotirić Akšić MM, Glavnik V, et al. Establishing the chromatographic fingerprints of flavan-3-ols and proanthocyanidins from rose hip (Rosa sp.) species. J Sep Sci. 2020;1-9. https://doi.org/10.1002/jssc.201901271 